\title{
Bioanalysis
}

\section{Methods and techniques for metabolic phenotyping}

\author{
“...metabolic phenotyping holds out the potential for great benefits \\ to both individuals and to society. If these benefits are to be realized, \\ then bioanalysis will be a key to their delivery."
}

First draft submitted: 18 October 2016; Accepted for publication: 21 October 2016; Published online: 6 December 2016

Keywords: endogenous metabolites $\bullet$ mass spectrometry $\bullet$ metabolomics $\bullet$ metabonomics - NMR spectroscopy

\section{"...based on the advances that we are seeing every day in this field, it seems self-evident that specific metabolic signatures that accu- rately predict an individual's propensity for developing a disease, or foretell their response to therapy, can only benefit research programs aimed at the delivery of precision medicine."}

Developments in 'global metabolic profiling', metabolomics [1] or metabonomics [2] to its adherents, undertaken with the aim of discovering new biomarkers that can be used to monitor and better understand the basic physiological processes (e.g., growth, aging and the body's reaction to stress such as exercise) or the development of disease (and response to treatment) continue apace. The aim of such studies is to obtain comprehensive metabolic profiles, containing as much biochemical information on the range and concentrations of endogenous metabolites as possible, as quickly as possible, in relevant samples such as biological fluids (e.g., plasma/serum and urine, etc.), tissues, exhaled breath, cell cultures and so on. The huge amounts of data gathered during these 'hypothesis-free' analyses are then subjected to advanced statistical analysis to determine which features of the metabolic phenotypes characterize the condition under study. The aim is to extract specific metabolites or groups of metabolites, that may represent potential biomarkers that are directly related to the biochemical changes associated with these biological states. From these initial hypothesis-free studies, the emerging metabolic data should result in the formulation of (testable) mechanistic hypotheses relating to the potential metabolic biomarkers to some underlying biochemistry that is directly reflected in the changes seen in the metabolome. Then, following on from these general metabolic phenotyping experiments, the use of more specific, targeted, approaches can be utilized to further investigate and validate the changes detected in the preceding studies, and begin to convert these potential biomarkers into useful practical biomarkers of the condition, rather than some unrelated general biochemical 'collateral damage' to the system under investigation. The advantages and limitations of targeted methods are ably discussed by Rosebrock in an editorial on the topic in this Special Focus Issue [3].

Clearly, obtaining these metabolic phenotypes, also known as 'metabotypes' [4], is not a trivial undertaking and requires advanced analytical techniques [5,6], rigorous quality control $[7,8]$ and appropriate methods for data analysis [9]. Currently, the main analytical technologies that are employed for metabotyping are centered on NMR (usually ${ }^{1} \mathrm{H}$ NMR) spectroscopy and MS. The techniques are often used in combination with each other as they provide complementary information. In the case of MS, analysis can be performed directly on the sample, using direct infusion MS (DIMS) methods, or the MS can be 'hyphenated' to a separation

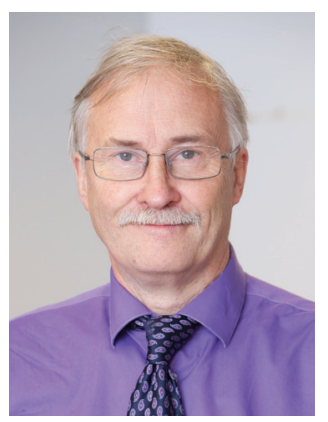

Ian Wilson

Section of Biomolecular Medicine, Division of Computational \& Systems Medicine, Department of Surgery \& Cancer, Faculty of Medicine, Imperial College, Exhibition Road, South Kensington, London SW7 2AZ, UK i.wilson@imperial.ac.uk 
technique. ${ }^{1} \mathrm{H}$ NMR spectroscopy remains popular for untargeted metabolic profiling because of its simplicity, broad metabolite detection capabilities, robustness and repeatability as well as the minimal need for sample preparation. DIMS is also valuable if, used with care and an awareness of its limitations, and its role in metabolomics is discussed in-depth in a review of the topic in this special issue [10]. Of the hyphenated MS techniques, GC-MS has by far the longest association with the general sample analysis of the sort practiced in metabotyping. However, except in the case of, for example, breath (or other) volatiles, the need for carefully optimized derivatization in order to modify otherwise involatile metabolites and facilitate their analysis by GC is a key requirement for success and this aspect is considered from a practical standpoint in another article in this issue [11]. The use of a targeted GC-MS method to obtain 'steroidomic' data on the cellular response of a range of steroid hormones to the in vitro exposure of $\mathrm{H} 295 \mathrm{R}$ cells (of proven value in screening for chemicals that affect steroid biosynthesis) to endocrine disruptors therefore provides a timely example of the application of this technology to gain insights into the changes wrought on cellular steroidogenic pathways by such chemicals [12].

Despite the continuing major role of GC-MS, it is increasingly clear that methods based on LC, such as HPLC-MS, and particularly U(H)PLC-MS, are set to dominate the field of metabolic profiling (both targeted and untargeted). The widespread availability of LC-MS systems together with the compatibility of, for example, reversed-phase separations with biological samples and the general lack of a requirement to derivatize analytes prior to analysis, have all led to its rapid take up.

Of course, when dealing with a specific problem the use of multiple analytical platforms, both for untargeted metabotyping and targeted investigations, is often required. An example of this approach is provided by the metabolite profiling of coelomic fluid and cells described in this special issue where four different techniques were employed. These included hydrophilic interaction liquid chromatography (HILIC)UHPLC-MS/MS, GC-MS, ${ }^{1} \mathrm{H}$ NMR spectroscopy and DIMS (using Fourier transform ion cyclotron ultra-high resolution MS [FT-ICR-MS]) [13]. Similarly two analytical platforms, GC-MS and LC-MS/MS, were employed for the targeted and untargeted metabolic profiling of feces and cecal tissue in a study of the effects of exercise and aging on the rat metabolome [14]. As a reminder that sometimes metabolites are also often associated with macromolecules an article on lipoprotein profiling methodology, based on the determination of apolipoprotein concentrations is also included in the articles collected in this special issue [15].
However, despite its general applicability and ease of use, LC-MS cannot yet be considered to provide a complete solution to the analysis of all classes of compounds. In particular profiling, polar ionic metabolites still provide a challenge, only partially solved by the application of HILIC-based separations and, while methods based on ion-pair chromatography currently provide the best LC-based solution [16-18], they bring with them problems associated with the contamination of the LC-MS system. So, while LC and GCMS-related methods dominate the other separation techniques used in metabolic profiling, other types of separation such as capillary electrophoresis (CE) may also be of benefit. Indeed CE provides a useful alternative to HILIC and ion pairing (IP)-LC-MS approaches for very polar ionic metabolites. As a result CE-MS has shown itself to be well suited to such 'difficult' analytes, and the advantages and applications of the technique for metabolomics are clearly described in a review article [19] in this special issue.

While the manuscripts presented in this Special Focus Issue highlight advances and successes in the application of advanced analytical chemical methods to the characterization of the complex mixtures of metabolites presented by biological samples, there remain problems that require resolution. Two of the elephants in the room that is metabolic phenotyping, which will certainly benefit from technological advances, are the ever present need for improved separations and rapid metabolite identification/confirmation of identity. In this respect ion mobility spectrometry, which provides both type of (very rapid) separation and physicochemical information via the associated collisional cross section, may well be of assistance. A timely perspective article on the use of ion mobility spectrometry and collisional cross-section data in the field of exposomics also forms a part of this special issue [20].

Thus, this special issue of Bioanalysis, focused as it is on the multianalyte measurement of endogenous metabolites, provides a collection of articles that cover many important aspects of the technologies used for the analysis of complex biological samples. They include authoritative general reviews, descriptions of research using both 'untargeted' metabotyping and examples of more specific targeted approaches, and also highlight potential new approaches.

As a result, the articles presented in this Special Focus Issue represent a 'snapshot' of both current applications and also advances that are occurring in the field of metabolic phenotyping. It is widely accepted that it is easy to predict the past but that predicting the future is fraught with danger. However, based on the advances that we are seeing every day in this field, it seems self-evident that specific metabolic signatures that accurately predict an 
individual's propensity for developing a disease, or foretell their response to therapy, can only benefit research programs aimed at the delivery of precision medicine. By providing a deeper understanding of the underlying biochemistry of the processes involved in health and disease, metabolic phenotyping holds out the potential for great benefits to both individuals and to society. If these benefits are to be realized, then bioanalysis will be a key to their delivery.

\section{References}

1 Fiehn O. Metabolomics - the link between genotypes and phenotypes. Plant. Mol. Biol. 48, 155-171 (2002).

2 Nicholson JK, Lindon JC, Holmes E. 'Metabonomics': understanding the metabolic responses of living systems to pathophysiological stimuli via multivariate statistical analysis of biological NMR spectroscopic data. Xenobiotica 29, 1181-1189 (1999).

3 Rosebrock AP. Targeted full-scan LC-MS metabolomics: simultaneous quantitation of knowns and feature discovery provides the best of both worlds. Bioanalysis 9(1), 5-8 (2016).

4 Gavaghan CL, Holmes E, Lenz E, Wilson ID, Nicholson JK. An NMR-based metabonomic approach to investigate the biochemical consequences of genetic strain differences: application to the C57BL10J and Alpk:ApfCD mouse. FEBS Lett. 484, 169-174 (2000).

5 Gika HG, Theodoridis GA, Plumb RS, Wilson ID. Current practice of liquid chromatography-mass spectrometry in metabolomics and metabonomics. J. Pharm. Biomed. Anal. 87, 12-25 (2014).

6 Zhang A, Sun H, Wang P, Han Y, Wang X. Modern analytical techniques in metabolomics analysis. Analyst 137 , 293-300 (2012).

7 Gika HG, Theodoridis GA, Wingate JE, Wilson ID. Within-day reproducibility of an HPLC-MS-based method for metabonomic analysis: application to human urine. $J$. Proteome Res. 6(8) 3291-3303 (2007).

8 Dunn WB, Wilson ID, Nicholls AW, Broadhurst D. The importance of experimental design and QC samples in large-scale and MS-driven untargeted metabolomic studies of humans. Bioanalysis 4, 2249-2264 (2012).

9 Alonso A, Marsal S, Julià A. Analytical methods in untargeted metabolomics: state of the art in 2015. Front. Bioeng. Biotechnol. 3, 23 (2015).

10 González-Domínguez R, Sayago A, Fernández-Recamales A. Direct infusion mass spectrometry for metabolomic phenotyping of diseases. Bioanalysis 9(1), 131-148 (2016).

11 Moros G, Chatziioannou AC, Gika HG, Raikos N, Theodoridis $\mathrm{G}$. Investigation of the derivatization conditions

\section{Financial \& competing interests disclosure}

The author has no relevant affiliations or financial involvement with any organization or entity with a financial interest in or financial conflict with the subject matter or materials discussed in the manuscript. This includes employment, consultancies, honoraria, stock ownership or options, expert testimony, grants or patents received or pending, or royalties.

No writing assistance was utilized in the production of this manuscript.

for GC-MS metabolomics of biological samples. Bioanalysis 9(1), 53-65 (2016).

12 Jumhawan U, Yamashita T, Ishida K, Fukusaki E, Bamba T. Simultaneous profiling of 17 steroid hormones for the evaluation of endocrine disrupting chemicals in H295R cells. Bioanalysis 9(1), 67-79 (2016).

13 Virgiliou C, Valianou L, Witting M et al. Metabolic profile of human coelomic fluid. Bioanalysis 9(1), 37-51 (2016).

14 Deda O, Gika H, Panagoulis T, Taitzoglou I, Raikos $\mathrm{N}$, Theodoridis G. Impact of exercise on fecal and cecal metabolome over aging. A longitudinal study in rats. Bioanalysis 9(1), 21-36 (2016).

15 Takeda H, Izumi Y, Tomita A et al. Lipoprotein profiling methodology based on determination of apolipoprotein concentration. Bioanalysis 9(1), 9-19 (2016).

16 Coulier L, Bas R, Jespersen S, Verheij E, van der Werf MJ, Hankemeier T. Simultaneous quantitative analysis of metabolites using ion-pair liquid chromatographyelectrospray ionization mass spectrometry. Anal. Chem. 78(18), 6573-6582 (2006).

17 Michopoulos F, Whalley N, Theodoridis G, Wilson ID, Dunkley TP, Critchlow SE. Targeted profiling of polar intracellular metabolites using ion-pair-high performance liquid chromatography and -ultra high performance liquid chromatography coupled to tandem mass spectrometry: applications to serum, urine and tissue extracts. $J$. Chromatogr. A 1349, 60-68 (2014).

$18 \mathrm{Lu}$ W, Clasquin MF, Melamud E, Amador-Noguez D, Caudy AA, Rabinowitz JD. Metabolomic analysis via reversed-phase ion-pairing liquid chromatography coupled to a stand alone Orbitrap mass spectrometer. Anal. Chem. 82, 3212-3221 (2010).

19 García A, Godzien J, Lopez-Gonzalves A, Barbas C. Capillary electrophoresis mass spectrometry as a tool for untargeted metabolomics. Bioanalysis 9(1), 99-130 (2016).

20 Metz TO, Baker ES, Schymanski EL et al. Integrating ion mobility spectrometry into mass spectrometry-based exposome measurements: what can it add and how far can it go? Bioanalysis 9(1), 81-98 (2016). 
